\title{
Carotid Stump as a Cause of Recurrent Embolism- Endovascular Treatment in a Case of Congenital Carotid Stump Syndrome
}

\author{
Jonghwa Shin, Jihoon Cha, ${ }^{\mathrm{b}}$ Pyoung Jeon, ${ }^{\mathrm{b}}$ Oh Young Bang ${ }^{\mathrm{a}}$ \\ Departments of Neurology and Radiology, Samsung Medical Center, Sungkyunkwan University, Seoul, Korea
}

Dear Sir:

Cryptogenic stroke, a stroke for which there is no specific attributable cause after a comprehensive evaluation for the most common causes, accounts for about one-fourth of stroke patients. Most cryptogenic strokes are thromboembolic and occur more frequently in younger patients. There have been efforts to detect paradoxical embolism in patients with cryptogenic embolic stroke because chronic anticoagulation can effectively prevent recurrent stroke in these patients. However, other causes may also cause cryptogenic embolic stroke, and the various causes of cryptogenic strokes have been increasingly recognized recently. ${ }^{1}$ Herein, we present a case of recurrent strokes possibly associated with a carotid stump related to congenital non-bifurcating common carotid artery (CCA) and its successful treatment using stenting.

A 39-year-old man was admitted to our hospital due to recurrent ischemic strokes. He had no risk factors for stroke except for smoking. He had a history of recurrent stroke despite the use of antithrombotics. Three years ago, he had a cortical infarction in the left anterior cerebral artery area with symptoms of transient right-sided weakness and was treated with cilostazol owing to an aspirin allergy (Figure 1). One year ago, he was admitted for transient right-sided weakness and motor aphasia that lasted 5 minutes. Magnetic resonance imaging (MRI) revealed an acute cortical infarction in the left middle cerebral artery (MCA) area (Figure 1). He was treated with warfarin temporarily, and was subsequently treated with clopidogrel.

While taking clopidogrel, the patient experienced a third ischemic stroke. He presented with sensory change in the right hand.
The neurologic exam result was normal, except for numbness in his right hand area. Diffusion-weighted imaging revealed an acute cortical infarction in the left MCA area and a chronic infarction in the left MCA and the anterior cerebral artery areas (Figure 1). Magnetic resonance angiography showed congenital agenesis of the left internal carotid artery (ICA) and an ICA stump. The left carotid area was supplied by a connection between the distal ICA and the external carotid artery (ECA) (Figure 1B). There was no stenotic lesion in the intracranial vessels and in the distal left common carotid artery of ECA origin. Carotid CT angiography did not show any atherosclerotic plaque in the carotid stump area. A selective left CCA injection revealed a bulbous carotid stump and turbulent flow within the ICA stump (Figure 2A). Comprehensive diagnostic work ups, including laboratory tests for coagulopathy, echocardiography with the bubble test to detect paradoxical embolism, 24-hour Holter monitoring, and coronary CT angiography for aortic pathology yielded negative results. Laboratory test for clopidogrel resistance was negative.

The decision was made to insert a stent and coil to block the stump because the patient suffered a recurrent left hemispheric stroke despite receiving antithrombotics, and only an ICA stump could be a possible source of the embolism. Stent insertion and stent-assisted coiling were successfully performed without complications (Figure 2B and C). A closed cell-type self-expanding stent (Wall stent, Boston Scientific, Ireland) was used. The patient was discharged with triflusal and clopidogrel treatment, and remained asymptomatic at 5 months of follow-up.

Carotid stump syndrome remains a rare entity, but it is a treatable cause of recurrent retinal and hemispheric symptoms after 

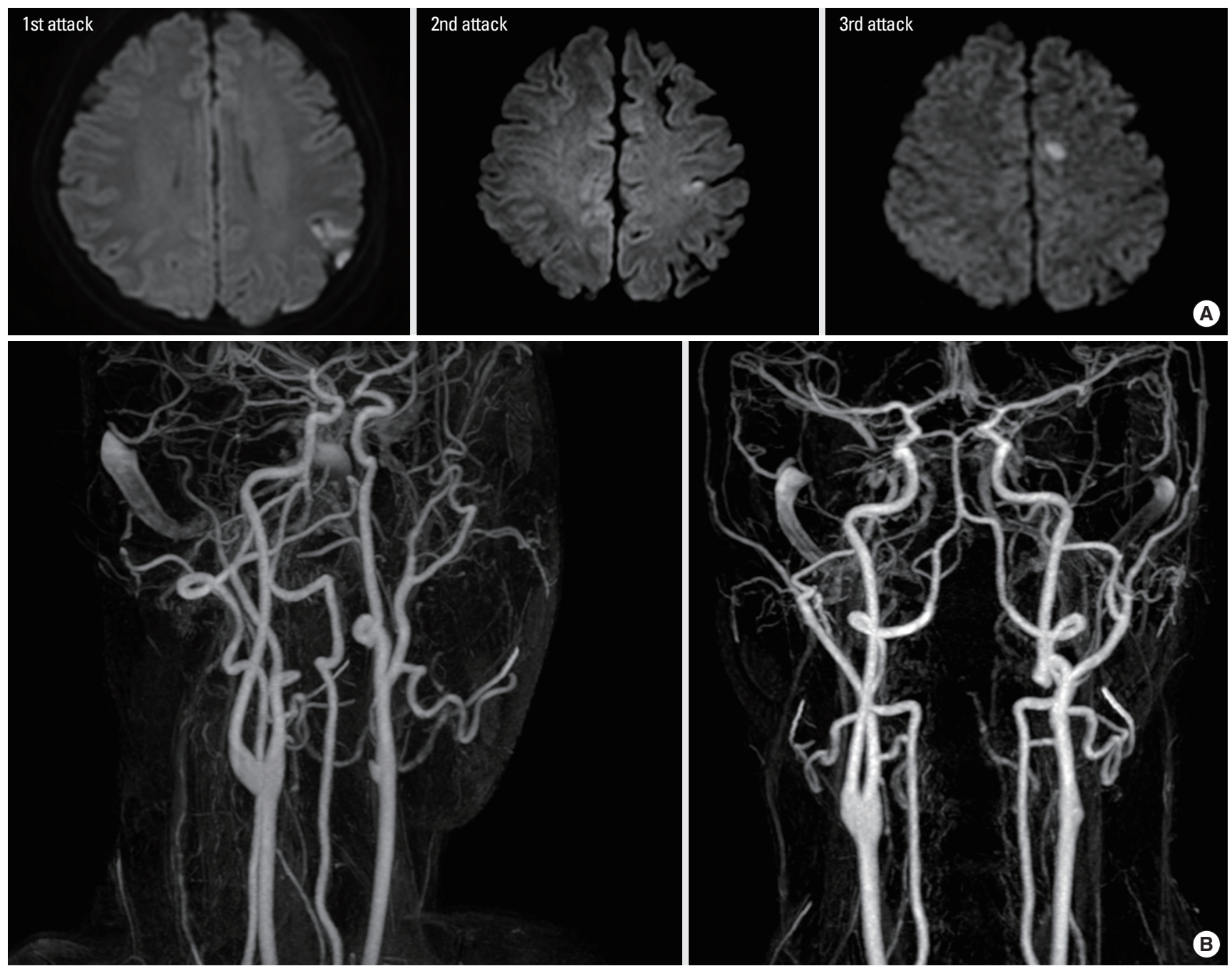

Figure 1. A total of three recurrent left hemispheric strokes occurred in 3 years. (A) Diffusion-weighted imaging findings of recurrent left middle or anterior cerebral artery area cortical infarcts. The patient first had a left anterior cerebral artery cortical infarction 3 years ago. A second ischemic stroke occurred in the left middle cerebral artery cortex a year later. A third ischemic stroke then occurred. (B) Magnetic resonance angiography findings.

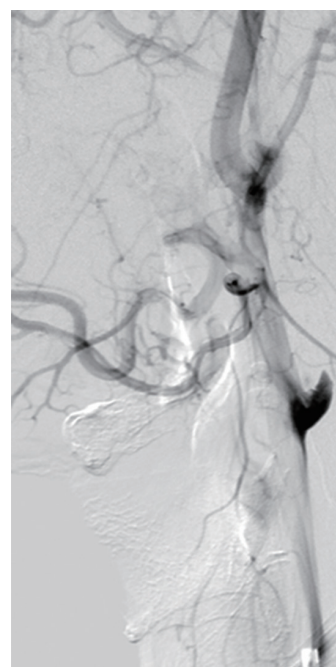

(A)

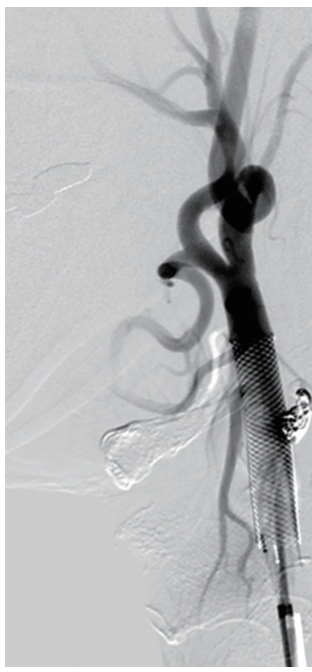

(B)

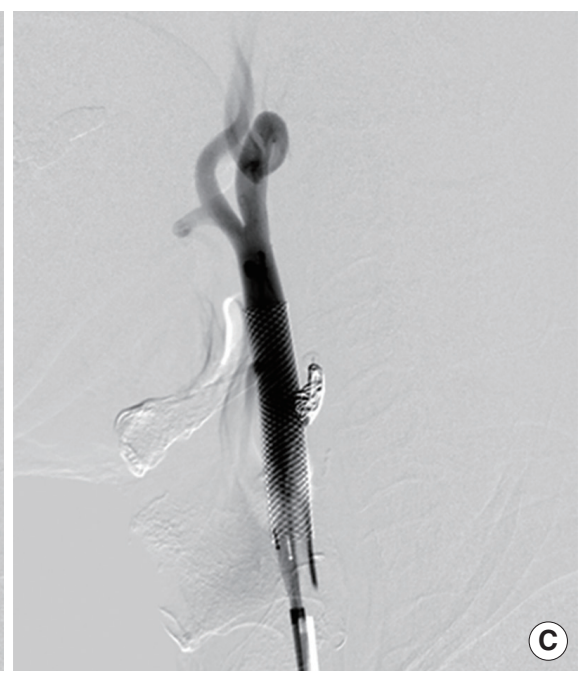

Figure 2. Conventional angiographic findings. (A) Cerebral angiography revealed turbulent flow in the left carotid stump. (B) Stenting and stent-assisted coiling were performed across the origin of the left internal carotid artery. (C) No residual stump on postprocedure angiography. 
ICA occlusion. Rarely, after complete occlusion of the ICA, a short patent portion of the proximal ICA can be shown by angiography as well as by other imaging modalities, and is referred to as the "carotid stump." In this case, congenital non-bifurcating CCA with an anomalous ECA-ICA connection, rather than acquired ICA occlusion could be the cause of the carotid stump. ${ }^{2}$

Both surgical exclusion of the carotid stump with endarterectomy of the ECA, and carotid stenting of the distal common and external carotid arteries to exclude the ICA stump, can be used. Lakshminarayan and colleagues reported the results of a small case series that used endovascular treatment suggesting that long-term outcomes are equivalent to those achieved by surgery. ${ }^{3}$ Our and other cases suggest that in the case of recurrent retinal or hemispheric symptoms with an ipsilateral carotid stump but no other demonstrable cause, carotid stenting with/without coiling should be considered to normalize the regional hemodynamics associated with the carotid stump.

\section{References}

1. Bang OY, Ovbiagele B, Kim JS. Evaluation of cryptogenic stroke with advanced diagnostic techniques. Stroke 2014;45: 1186-1194.

2. Kim CH, Cho YD, Jung SC, Ahn JH, Kang HS, Kim JE, et al. Anomalous external carotid artery-internal carotid artery anastomosis in two patients with proximal internal carotid arterial remnants. Korean J Radiol 2015;16:914-918.

3. Lakshminarayan R, Scott PM, Robinson GJ, Ettles DF. Carotid stump syndrome: Pathophysiology and endovascular treatment options. Cardiovascular and interventional radiology 2011; 34(Suppl 2):S48-52.

Correspondence: Oh Young Bang

Department of Neurology, Samsung Medical Center, Sungkyunkwan University, 81 Irwon-ro, Gangnam-gu, Seoul 06351, Korea

Tel: +82-2-3410-3599, Fax: +82-2-3410-0052

E-mail: ohyoung.bang@samsung.com

Received: May 21, 2015

Revised: June 15, 2015

Accepted: June 16, 2015

The authors have no financial conflicts of interest. 